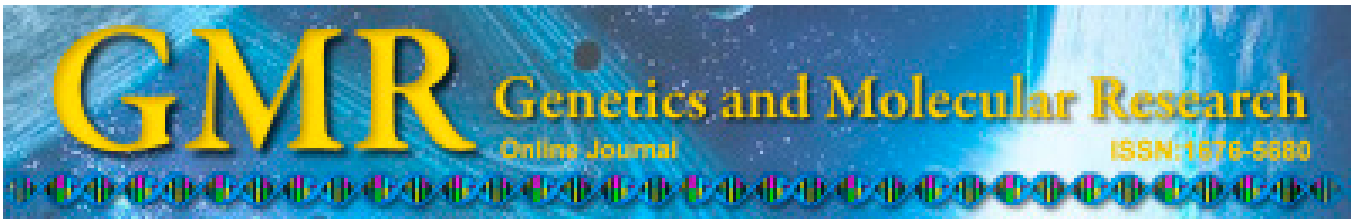

\title{
Analysis of quantitative trait loci for main plant traits in soybean
}

\author{
D. Yao*, Z.Z. Liu*, J. Zhang, S.Y. Liu, J. Qu, S.Y. Guan, L.D. Pan, \\ D. Wang, J.W. Liu and P.W. Wang \\ Biotechnology Center, Jilin Agricultural University, Changchun, China \\ *These authors contributed equally to this study. \\ Corresponding author: P.W. Wang \\ E-mail: davidmedsci@sina.com
}

Genet. Mol. Res. 14 (2): 6101-6109 (2015)

Received June 30, 2014

Accepted November 19, 2014

Published June 8, 2015

DOI http://dx.doi.org/10.4238/2015.June.8.8

\begin{abstract}
Plant traits are important indices for regulating and controlling yield ability in soybean varieties. It is important to comprehensively study the quantitative trait locus (QTL) mapping for soybean plant traits, cloning related genes, and marker assistant breeding. In this study, $236 \mathrm{~F}_{2}$ generation plants and a derivative group were constructed by using Jiyu50 and Jinong18, obtained from Jilin Province. A total of 102 simple sequence repeat markers were used to construct a genetic linkage map. With 2 years of molecular and phenotypic data, QTL analyses and mapping were conducted for soybean maturity, plant height, main stem node, main stem branch, seed weight per plant, and more. Five main plant traits were analyzed via inclusive composite interval mapping using QTL IciMapping v2.2. Using one-dimensional scanning, a total of 30 QTLs were detected and distributed across 1 (A1), 4 (C2), and $12(\mathrm{G})$. There were 9 linkage groups, including 16 major QTLs. Using two-dimensional scanning, 7 pairs of epistatic QTL interactions for maturity and plant height were detected in the soybean.
\end{abstract}

Key words: Soybean; Plant trait; SSR; Quantitative trait loci; Inclusive composite interval mapping 


\section{INTRODUCTION}

The main plant traits of the soybean plant, such as maturity, plant height, and number of main stem nodes, etc., comprise an important foundation for increasing soybean variety yield ability to full play. In general, it is thought that the main soybean plant traits present quantitative trait inheritance, influenced by genetic effects and environmental factors. For traditional, high-yield breeding of soybean, breeding selection is conducted mainly in terms of individual phenotype. Thus, breeding efficiency is low, and the breeding cycle is time consuming. Therefore, it is very difficult to select these traits using only traditional breeding methods (Wang, 2005). With the development of molecular genetics and emergence of molecular marker technology, such as restricted fragment length polymorphisms, random amplification polymorphism DNA, simple sequence repeat, and amplified fragment length polymorphism, it is feasible to selectively breed the target traits via marker-assisted breeding, especially for the construction of a high-density soybean genetic map, which would greatly promote the development of breeding (Fang et al., 2002).

Since the first report of soybean quantitative trait locus loci (QTL) in 1990 (Keim et al., 1990), molecular marker technology has undergone rapid development. There are additional studies on soybean yield, quality, and morphology traits. According to the data published in SoyBase (http:://www.Soybase.org), 173 soybean plant height QTLs have been currently mapped, which were distributed into many linkage groups, including A2, B1, C1, D1, E, F, H, J, and L (Lee et al., 1996; Mansur et al., 1996; Mian et al., 1998). Except for the D2 and E linkage groups, reported maturity-related QTLs were distributed across 18 additional linkage groups, predominantly the $\mathrm{C} 2, \mathrm{~L}$, and $\mathrm{M}$ linkage groups. Fine mapping and map-based cloning have been conducted for some QTLs (Yamanaka et al., 2005; Watanabe et al., 2009; Su et al., 2010). There are fewer studies on soybean branch trait loci, and the genetic contribution ratios of mapped soybean branch-related QTLs is low. Only several contribution ratios are $>20 \%$ locus. These include one QTL with a contribution ratio of 50.9\%, reported by Guan (2004) in the A1 linkage group, and 2 QTLs with contribution ratios of 20.9 and $22.1 \%$, respectively, which were mapped in 2009 in the I and L linkage groups (Zhou et al., 2009).

In this study, the $\mathrm{F}_{2}$ segregation group following hybridization (i.e., by crossing Jiyu 50 and Jinong 18, with the greater variation in soybean main plant traits) was used as the experimental material. Then, QTL mapping and an effect analysis were conducted for 5 main plant traits (i.e., soybean maturity, plant height, number of main stem nodes, number of main stem branches, and seed number per plant) by inclusive composite interval mapping (ICIM) via QTL IciMapping v2.2 for 2 continuous years in order to provide references for soybean yield-related basic research and a theoretical basis for breeding novel high-yield varieties of soybean via molecular marker-assisted selection and cloning of the relevant gene.

\section{MATERIAL AND METHODS}

\section{Materials}

\section{Construction of the soybean segregation group}

Soybean variety Jiyu 50 (i.e., female parent) was hybridized with Jinong 18 (i.e., male 
parent) in the experimental field of Jilin Agricultural University in the summer of 2005 to obtain the $\mathrm{F}_{0}$ generation. In May 2007, an $\mathrm{F}_{2}$ individual plant was obtained. In May 2008, an $\mathrm{F}_{3}$ plant was obtained from 236 individual plants from the $\mathrm{F}_{2}$ generation. A randomized block design was used for the field experiment. The length and width of each line were 4.5 and 0.4 $\mathrm{m}$, respectively; 50 seeds were planted in every line, and every experiment was repeated 3 times. For each plant row, 10 plants were randomly harvested for the indoor seed test.

\section{Primers}

Primers were selected according to the soybean "public map" (2003), and 380 pairs of SSR primers were primarily confirmed from the soybean database SoyBase (http://soybase. agron.iastate.edu) and synthesized by Beijing Sunbiotech Co., Ltd. (Beijing, China).

\section{DNA extraction and SSR analyses}

Genomic DNA of the soybean was isolated from the leaf tissue by the improved SDS method (Wang and Fang, 2002). Polymerase chain reaction (PCR) was performed in a $15-\mu \mathrm{L}$ volume containing $0.6 \mu \mathrm{L}$ genomic DNA $(50 \mathrm{ng} / \mu \mathrm{L}), 0.6 \mu \mathrm{L}$ dNTP mixtures $(10 \mathrm{mM}), 0.6 \mu \mathrm{L}$ SSR primer $(25 \mu \mathrm{M}), 1.5 \mu \mathrm{L} 10 \mathrm{X}$ PCR buffer $\left(\right.$ contain $\left.\mathrm{Mg}^{2+}\right), 0.15 \mu \mathrm{L}$ Taq polymerase $(5 \mathrm{U} /$ $\mu \mathrm{L}$ ), and $10.95 \mu \mathrm{L}$ double-distilled water. The PCR conditions were 5 min at $94^{\circ} \mathrm{C}$; followed by 40 cycles of $30 \mathrm{~s}$ at $94^{\circ} \mathrm{C}, 30 \mathrm{~s}$ at $50^{\circ} \mathrm{C}$, and $30 \mathrm{~s}$ at $72^{\circ} \mathrm{C}$; then $8 \mathrm{~min}$ at $72^{\circ} \mathrm{C}$. After amplification, the PCR products were mixed with a loading buffer, denatured for $5 \mathrm{~min}$ at $94^{\circ} \mathrm{C}$, and kept at $0^{\circ} \mathrm{C}$. The denatured PCR products were separated on $8 \%(\mathrm{w} / \mathrm{v})$ denaturing polyacrylamide gel and visualized by silver staining (Sanguinetti et al., 1994).

\section{Construction of the molecular genetic map}

The Mapmaker Exp3.0 software (The Whitehead Institute for Biomedical Research, Massachusetts Institute of Technology, Cambridge, MA, USA) was used for map construction (Wang, 2009). Inter-marker linkage analysis and grouping were conducted by the "Group" command. For the number of linked markers $<8$, sorting was conducted by the "Compare" command, and sorting was conducted by the "Ripple" command for the number of linked markers $>8$ (Schneider et al., 1997; Liu et al., 2000). The error detection level was set at 1\%, and the recombination rate was converted to genetic distance $(\mathrm{cM})$ by use of the Rosambi function. The WinQTLCart2.5 software (Bioinformatics Research Center, North Carolina State University, Raleigh, NC, USA) was used for drawing the genetic linkage map.

\section{Testing of main plant traits of soybean}

An indoor seed test and statistics were carried out for maturity, plant height, number of main stem nodes, number of main stem branches, and seed number per plant of Jiyu 50, Jinong 18, the $\mathrm{F}_{2}$ generation, and their derivative groups from 2007 to 2008.

\section{SSR data record and statistics analysis}

Electrophoresis strips following SSR-PCR were recorded; strips matching the female 
parent were marked as " 2 ", heterozygous strips were marked as " 1 ", strips matching the male parent were marked as " 0 ", and missing strips were marked as "- 1 ".

\section{QTL mapping}

ICIM was conducted for maturity, plant height, number of main stem nodes, number of main stem branches, and seed number per plant of the $\mathrm{F}_{2}$ and $\mathrm{F}_{3}$ segregation groups by the QTL IciMapping v2.2 software (Institute of Crop Science, Chinese Academy of Agricultural Sciences CAAS, Beijing, China); the scanning step was $1.0 \mathrm{cM}$. In case of marker stepwise regression, probability levels of variables in and out of the model were 0.01 and 0.02 , respectively. An LOD value of $2.5(L R=12.075)$ was set as the threshold value for QTL mapping and effect estimation.

\section{RESULTS}

\section{Performance of main plant traits of soybean in the $F_{2}$ segregation group}

The indoor seed tests and analyses were conducted for 5 plant traits (i.e., maturity, plant height, number of main stem nodes, number of main stem branches, and seed number per plant) of 236 individual plants in the $\mathrm{F}_{2}$ group and their parents. Results showed that there were significant differences in various traits between the 2 parents, and the mean of the $\mathrm{F}_{2}$ group was greater than the parents, suggesting that all 5 traits exhibited transgressive inheritance (Table 1). Moreover, frequency distribution, skewness test, and kurtosis test of the main plant traits suggested that all 5 plant trait indicators such as maturity, plant height, number of main stem nodes, number of main stem branches, and seed number per plant presented normal distributions and smaller skewness values; the skewness values of both plant height and the number of main stem nodes were negative and exhibited a left-skewed distribution; skewness values of maturity, number of main stem branches, and seed number per plant were positive and exhibited a right-skewed distribution. Kurtosis of various agronomic traits was more flat and belonged to platykurtosis. Overall, all main group plant traits exhibited normal distributions and had a wide range of distribution frequencies, thus exhibiting the typical quantitative genetic model.

Table 1. Distribution of main plant traits of soybean in the parent and $\mathrm{F}_{2}$ generations.

\begin{tabular}{|c|c|c|c|c|c|c|c|c|}
\hline \multirow[t]{2}{*}{ Traits } & \multicolumn{2}{|c|}{ Parents } & \multicolumn{6}{|c|}{$\mathrm{F}_{2}$} \\
\hline & Jiyu 50 & Jinong 18 & Average value & Standard variance & Max & Min & Skewness & Kurtosis \\
\hline Maturity (days) & 134 & 124 & 128 & 4.47 & 140 & 118 & 0.27 & -0.78 \\
\hline Plant height $(\mathrm{cm})$ & 109.01 & 88.3 & 95.15 & 14.66 & 138.00 & 40.00 & -0.15 & 0.71 \\
\hline Numbers of main stem nodes & 22.6 & 19 & 20.66 & 3.01 & 29 & 11 & -0.46 & 0.53 \\
\hline Number of main stem branches & 2.5 & 4.3 & 2.01 & 1.01 & 6 & 0 & 0.82 & 0.77 \\
\hline Seed weight per plant (g) & 35.65 & 23.61 & 32.28 & 14.63 & 105.21 & 10.19 & 0.84 & 0.87 \\
\hline
\end{tabular}

\section{Construction of a genetic map of soybean}

In this study, 380 pairs of SSR primers were assessed between male and female parents to carry out polymorphic primer screening. Among them, 118 pairs of primers 
exhibited better polymorphic loci between parent DNAs, and the polymorphic percentage was $31.05 \%$. Subsequently, PCR amplification was conducted for 118 pairs of SSR primers exhibiting better polymorphism between male and female parent DNAs on 236 individual plants in the $\mathrm{F}_{2}$ segregation group. After testing via $8 \%$ denaturing polyacrylamide gel electrophoresis, statistics was conducted to obtain spectral band information. The data file was called out by Mapmaker Exp3.0. As recombination rate $\leq 0.50$, possible linkage groups were speculated. One group containing $\geq 2$ markers was defined as one linkage group. Finally, 102 SSR markers were classified into 25 genetic linkage groups. In addition, 16 SSR markers were not integrated into linkage groups. Ultimately, one soybean SSR genetic linkage map containing 102 markers was constructed (Figure 1). The total length of this map was $1380.3 \mathrm{cM}$, and the average distance between the markers was $13.53 \mathrm{cM}$. The average length of each linkage group was $55.2 \mathrm{cM}$, and the marker number of each linkage group was 4.0.
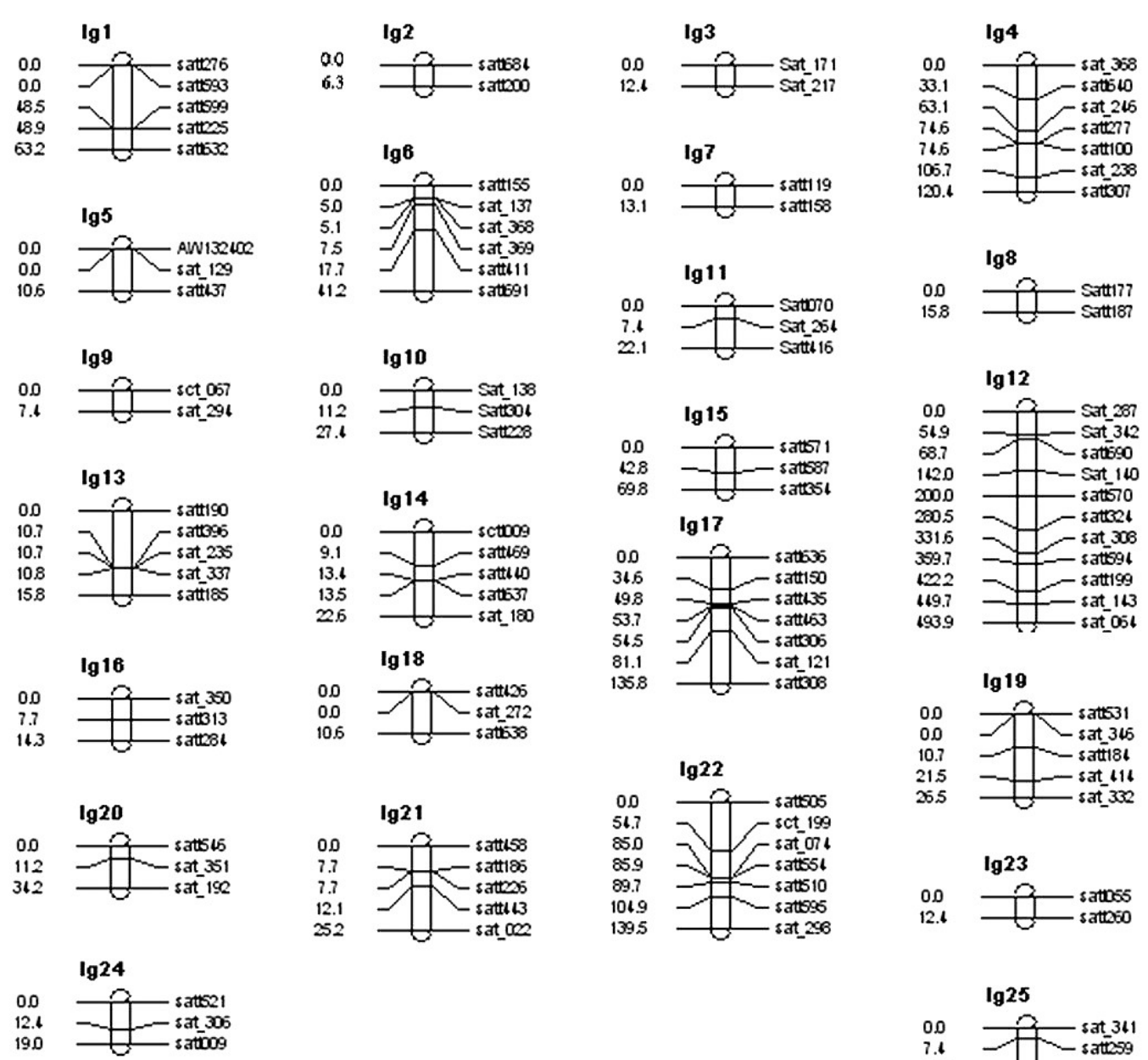

Figure 1. Soybean molecular genetic map based on simple sequence repeat markers.

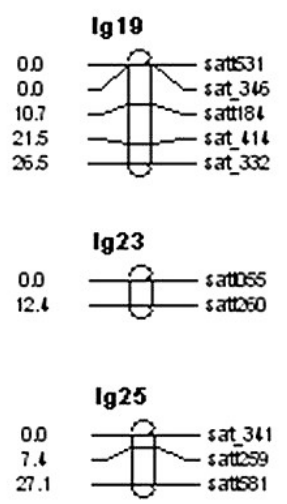




\section{QTL mapping and analysis of main plant traits of soybean}

When one-dimensional scanning (ICIM-ADD) was conducted for 5 main plant traits (i.e., soybean maturity, plant height, number of main stem nodes, number of main stem branches, and seed weight per plant) by ICIM, with LOD $=2.5$ as the threshold value for 2 continuous years, a total of 30 QTLs with obvious additive effects were detected throughout 9 genetic linkage groups, including 16 QTLs with main effects (Table 2).

\begin{tabular}{|c|c|c|c|c|c|c|c|c|}
\hline Traits & Years & $\begin{array}{l}\text { Linkage } \\
\text { group }\end{array}$ & QTL & Marker flanking & $\begin{array}{l}\text { QTL position } \\
\text { (front /rear; cM) }\end{array}$ & $\begin{array}{l}\text { LOD } \\
\text { value }\end{array}$ & $\mathrm{AE}$ & PVE (\%) \\
\hline \multirow[t]{7}{*}{ Maturity } & 2007 & $4(\mathrm{C} 2)$ & $q S M-4-1$ & Sat_246-Satt277 & $10.9 / 0.6$ & 3.83 & -2.49 & 7.48 \\
\hline & 2008 & $4(\mathrm{C} 2)$ & $q S M-4-1$ & Satt100-Sat_238 & $16.4 / 15.7$ & 4.29 & -3.06 & 17.65 \\
\hline & & & $q S M-4-2$ & Sat_238-Satt307 & $3.3 / 10.4$ & 2.69 & -1.55 & 5.40 \\
\hline & & $6(\mathrm{~A} 1)$ & $q S M-6-1$ & Satt411-Satt691 & $11.3 / 12.2$ & 2.60 & 1.45 & 4.88 \\
\hline & & $12(\mathrm{G})$ & $q S M-12-1$ & Satt594-Satt199 & $37.3 / 25$ & 7.32 & 1.76 & 50.51 \\
\hline & & $17(\mathrm{M})$ & $q S M-17-1$ & Sat_121-Satt308 & $29.9 / 24.8$ & 2.66 & -0.68 & 4.95 \\
\hline & & $22(\mathrm{~F})$ & $q S M-22-1$ & Satt505-Sct_199 & $30.0 / 24.7$ & 2.65 & -0.67 & 5.14 \\
\hline \multirow[t]{9}{*}{ Plant height } & 2008 & 3 (A1) & $q P H-3-1$ & Sat_171-Sat_217 & $8.0 / 4.4$ & 2.66 & -1.96 & 4.36 \\
\hline & & $4(\mathrm{C} 2)$ & $q P H-4-1$ & Sat_368-Satt640 & $9.0 / 24.1$ & 2.60 & -1.39 & 7.99 \\
\hline & & $12(\mathrm{G})$ & $q P H-12-1$ & Satt324-Sat_308 & $33.5 / 17.6$ & 2.87 & -4.87 & 14.92 \\
\hline & & $17(\mathrm{M})$ & $q P H-17-1$ & Satt636-Satt150 & $18.0 / 16.6$ & 2.59 & 2.71 & 4.56 \\
\hline & & & $q P H-17-2$ & Satt306-Satt_121 & $12.5 / 14.4$ & 3.17 & 3.63 & 6.22 \\
\hline & & & $q P H-17-3$ & Satt_121-Satt308 & $19.6 / 34.8$ & 2.71 & 2.74 & 4.10 \\
\hline & & $22(\mathrm{~F})$ & $q P H-22-1$ & Satt505-Sct_199 & $20.0 / 34.7$ & 2.70 & 2.73 & 4.06 \\
\hline & & & $q P H-22-2$ & Satt595-Sat_298 & $16.1 / 18.5$ & 2.66 & 2.80 & 4.75 \\
\hline & & $23(\mathrm{~K})$ & $q P H-23-1$ & Satt055-Satt260 & $8.0 / 4.4$ & 2.66 & -1.96 & 4.36 \\
\hline Numbers of main stem nodes & 2007 & $15(\mathrm{I})$ & $q N M S N-15-1$ & Satt571-Satt587 & $22.0 / 20.8$ & 2.61 & -1.78 & 30.80 \\
\hline \multirow[t]{4}{*}{ Numbers of main stem branches } & 2007 & $1(\mathrm{~A} 1)$ & $q N V B-1-1$ & Satt593-Satt599 & $23.5 / 25.0$ & 2.75 & 0.64 & 20.81 \\
\hline & & $12(\mathrm{G})$ & $q N V B-12-1$ & Sat_287-Sat_342 & $23.0 / 31.9$ & 4.02 & 0.73 & 25.38 \\
\hline & & & $q N V B-12-2$ & Satt690-Sat_140 & $45.3 / 28.0$ & 7.23 & 0.79 & 31.83 \\
\hline & & & $q N V B-12-3$ & Sat_140-Satt570 & $21.0 / 37.0$ & 6.13 & 0.79 & 29.13 \\
\hline \multirow[t]{9}{*}{ Seed weight per plant } & 2007 & $4(\mathrm{C} 2)$ & $q S W P P-4-1$ & Satt640-Sat_246 & $18.9 / 11.1$ & 8.51 & 14.74 & 29.21 \\
\hline & & $12(\mathrm{G})$ & $q S W P P-12-1$ & Satt690-Sat_140 & $47.3 / 26$ & 6.60 & 14.63 & 29.37 \\
\hline & & & $q S W P P-12-2$ & Sat_140-Satt570 & $18.0 / 40.0$ & 3.82 & 10.96 & 13.69 \\
\hline & & & $q S W P P-12-3$ & Satt570-Satt324 & $28.0 / 52.5$ & 5.76 & -15.73 & 30.92 \\
\hline & & & $q S W P P-12-4$ & Satt324-Sat_308 & $22.5 / 28.6$ & 3.57 & 11.45 & 14.42 \\
\hline & & & $q S W P P-12-5$ & Sat_308-Satt594 & $17.4 / 10.7$ & 4.84 & -16.55 & 18.98 \\
\hline & & & $q S W P P-12-6$ & Satt594-Satt199 & $21.3 / 41.2$ & 6.83 & -16.40 & 26.02 \\
\hline & & & $q S W P P-12-7$ & Sat_143-Sat_064 & $23.3 / 20.9$ & 4.78 & 14.51 & 25.25 \\
\hline & 2008 & $17(\mathrm{M})$ & $q S W P P-17-1$ & Sat306-Sat_信21 & $12.5 / 14.1$ & 2.76 & -0.28 & 4.27 \\
\hline
\end{tabular}

The linkage group in capital letters indicates the public genetic map of the linkage group number; QTL position (front/rear): "Front" is the first marker distance away from the marker interval, and "rear" is the second marker distance away from the marker interval. A new QTL nomenclature was established by the American Soybean Genetic Association in 2004. AE = additive effect; PVE = phenotypic variation explained.

Seven detected QTLs were relevant to soybean maturity and distributed across 6 linkage groups, including 4 (C2), 6 (A1), 12 (G), 17 (M), 20 (D1b), and 22 (F); LOD values ranged from 2.65 to 7.32 , and the phenotypic variation rate was between 4.95 and $50.51 \%$. Among the 7 detected QTLs relevant to soybean maturity, 2 QTLs (i.e., $q S M-4$ 14 and $q S M-4-2)$ were detected in 2008 and presented a distribution of clusters on the 4 (C2) linkage group.

Ten detected QTLs were relevant to soybean plant height and distributed across 6 linkage groups, including 3 (A1), $4(\mathrm{C} 2), 12(\mathrm{G}), 17(\mathrm{M}), 22(\mathrm{~F})$, and $23(\mathrm{~K})$; LOD values ranged 
from 2.59 to 3.17 , and the phenotypic variation rate was between 4.06 and $14.92 \%$. Among these 10 QTLs, 3 (i.e., $q P H-17-1, q P H-17-2$, and $q P H-17-3$ ) presented a distribution of clusters on the $17(\mathrm{M})$ linkage group.

In 2007, one main-effect QTL relevant to the number of main stem nodes of soybean was detected on the 15 (I) linkage group, and the phenotypic variation rate was $30.80 \%$. A total of 4 QTLs relevant to the number of main stem branches of soybean were detected and distributed on the 1 (A1) and 12 (G) linkage groups. LOD values ranged from 2.75 to 7.23 , and the phenotypic variation rate was between 20.81 and $31.83 \%$. Among the 4 QTLs, 2 (i.e., $q N V B-12-2$ and $q N V B-12-2$ ) presented distribution clusters on the $12(\mathrm{G})$ linkage group.

A total of 9 QTLs relevant to seed weight per plant of soybean were detected and distributed across 3 linkage groups, including 4 (C2), 12 (G), and $17(\mathrm{M})$. LOD values ranged from 2.76 to 8.51 , and the phenotypic variation rate was between 4.27 and $30.92 \%$. Among these 9 QTLs, 6 (i.e., qSWPP-12-1, qSWPP-12-2, qSWPP-12-3, qSWPP-12-4, $q S W P P-12-5$, and $q S W P P-12-6)$ detected in 2007 presented distribution clusters on the 12 (G) linkage group.

When two-dimensional scanning (ICIM-EPI) was conducted for 5 main plant traits (i.e., soybean maturity, plant height, number of main stem nodes, number of main stem branches, and seed weight per plant) by ICIM, with LOD $=2.5$ as the threshold value, a total of 7 pairs of epistatic interacting QTLs were detected and distributed across 7 genetic linkage groups (Table 3).

In 2008, a total of 3 pairs of epistatic interacting QTLs influencing soybean maturity were detected among the different linkage groups. LODAA (variation caused only by measuring epistasis) values ranged from 2.58 to 2.73 , and the explicable phenotypic variation rate was between 1.09 and $5.41 \%$. In 2008, a total of 4 pairs of epistatic interacting QTLs influencing soybean plant height were detected among the different linkage groups. LODAA values ranged from 2.54 to 2.64. Among them, the maximum phenotypic variation rate of $q P H-3-1 \times q P H-22-2$ on the $3(\mathrm{~A} 1)$ and 22 (F) linkage groups was $9.79 \%$, and the minimum phenotypic variation rate of $q P H-3-1 \mathrm{x} q P H-17-1$ on the 3 (A1) and 17

Table 3. Epistatic interacting QTLs of soybean main plant traits detected by the ICIM-EPI method.
\begin{tabular}{lllllllc}
\hline Traits & Years & QTL1 & Interval & QTL2 & Interval & LODAA & PVE\% \\
\hline Maturity & 2008 & $q S M-17-1$ & Satt306-Sat_121 & $q S M-4-1$ & Satt100-Sat_238 & 2.69 & 5.41 \\
& & $q S M-22-1$ & Satt505-Sct_199 & $q S M-4-1$ & Satt100-Sat_238 & 2.73 & 5.37 \\
\multirow{5}{*}{ Plant height } & \multirow{2}{*}{2008} & $q S M-22-1$ & Satt505-Sct_199 & $q S M-12-1$ & Satt594-Satt199 & 2.58 & 1.09 \\
& & $q P H-3-1$ & Sat_171-Sat_217 & $q P H-17-1$ & Satt636-Satt150 & 2.60 & 8.12 \\
& & $q P H-3-1$ & Sat_171-Sat_217 & $q P H-22-2$ & Sct_199-Sat_074 & 2.64 & 9.79 \\
& & $q P H-17-2$ & Satt306-Satt_121 & $q P H-23-1$ & Satt055-Satt260 & 2.59 & 8.15 \\
& & $q P H-22-1$ & Satt505-Sct_199 & $q P H-23-1$ & Satt055-Satt260 & 2.54 & 9.76 \\
\hline
\end{tabular}

$\mathrm{PVE}=$ phenotypic variation explained.

(M) linkage groups was $8.12 \%$.

\section{DISCUSSION}

In this study, a QTL analysis was conducted for 5 main plant traits (i.e., soybean maturity, plant height, number of main stem nodes, number of main stem branches, and seed 
weight per plant) by the genetic linkage map constructed over 2 continuous years, and a total of 30 QTLs with additive effects were detected; however, these QTLs were not evenly distributed across the linkage groups. Some linkage groups contained more QTLs, while others contained limited or no QTLs. According to the mapping results, most QTLs of the relevant traits gathered in similar areas of the chromosomes; the phenomenon of distribution of clusters occurring in genes with relevant functions was popularly present. For example, it was found that genes controlling seed weight per plant and the number of main stem branches presented an obvious distribution of clusters on the $\mathrm{G}$ linkage group. These function-relevant genes were distributed in clusters in the same genome region and in the same environment as chromatin; an explanation is that function-relevant gene expressions all occur during a certain period of individual development, which is closely related to the developmental stage.

According to a comparison of domestic and overseas studies, all plant traits such as maturity, plant height, and the number of main stem nodes exhibit consistent correspondence. In the present study, it was found that maturity QTLs were on the A1, C2, F, G, and M linkage groups in the public genetic map, which is nearly in line with the soybean maturity-relevant QTL detection results previously reported (Sun et al., 2009). In the present study, plant heightrelevant QTLs were distributed on the A1, C2, G, F, M, and K linkage groups in the public genetic map. Among them, a QTL on the C2 linkage group was more similar to the result reported by Wu et al. (2001).

The QTL study suggests that the epistatic effect plays an important role in quantitative traits (Orf et al., 1999; Specht et al., 2001), and there are few studies on soybean agronomy trait epistasis. In the present study, when two-dimensional scanning was conducted via ICIM, 7 pairs of epistatic interacting QTLs occurring among 5 linkage groups were detected for 2 plant traits (i.e., soybean maturity and plant height). Among the 7 pairs of epistatic interacting QTLs, none occurred on the same linkage group; on different linkage groups, there was oneto-one interaction, and, on occasion, one QTL simultaneously interacted with many QTLs, thus suggesting the universality and complexity of the epistatic interaction. Therefore, it is of great importance for research on the genetic breeding of soybean to further investigate epistatic QTLs with large contribution ratios.

\section{Conflicts of interest}

The authors declare no conflict of interest.

\section{ACKNOWLEDGMENTS}

Research supported by the Agency of Science and Technology of Jilin Provincial Science \& Technology Department (\#20140204021NY, \#20140101015JC) and the Campus Startup Funds of Jilin Agricultural University (\#201242).

\section{REFERENCES}

Fang XJ, Wu WR and Tang JL (2002). DNA marker assisted breeding of plant. Science Press, Beijing (in Chinese). Guan RX (2004). QTL mapping of soybean agronomic characters and genetic diversity analysis of soybean cultivars from China and Japan. Doctoral thesis, Chinese Academy of Agricultural Sciences (CAAS), Beijing (Abstract in English).

Keim P, Diers BW, Olson TC and Schoemaker RC (1990). RFLP mapping in soybean: association between marker loci and variation in quantitative traits. Genetics 126: 735-742. 
Lee SH, Bailey MA, Mian MA, Shipe ER, et al. (1996). Identification of quantitative trait loci for plant height, lodging, and maturity in a soybean population segregating for growth habit. Theor. Appl. Genet. 92: 516-523.

Liu F, Zhuang BC, Zhang JS and Chen SY (2000). Construction and analysis of soybean genetic map. Acta Genet. Sin. 27: 1018-1026.

Mansur LM, Orf JH, Chase K, Jarvik T, et al. (1996). Genetic mapping of agronomic traits using recombinant inbred lines of soybean. Crop. Sci. 36: 1327-1336.

Mian MAR, Ashley DA, Vencill WK and Boerma HR (1998). QTLs conditioning early growth in a soybean population segregating for growth habit. Theor. Appl. Genet. 97: 1210-1216.

Orf JH, Chase K, Jarvik T, Mansur LM, et al. (1999). Genetics of soybean agronomic traits: I. Comparison of three related recombinant inbred populations. Crop Sci. 39: 1642-1651.

Sanguinetti CJ, Dias Neto E and Simpson AJ (1994). Rapid silver staining and recovery of PCR products separated polyacrylamide gels. Biotechniques 17: 914-921.

Schneider KA, Brothers ME and Kelly JD (1997). Marker assisted selection to improve drought resistance in common bean. Crop Sci. 37: 51-60.

Specht JE, Chase K, Macrander M, Graef GL, et al. (2001). Soybean response to water: A QTL analysis of drought tolerance. Crop Sci. 41: 493-509.

Su CF, Lu WG, Zhao TJ and Gai JY (2010). Verification and fine-mapping of QTLs conferring days to flowering in soybean using residual heterozygous lines. Chin. Sci. Bull. 55: 332-341.

Sun DS, Li WB, Zhang ZC and Chen QS (2009). Analysis of QTL for growth period in soybean. Soybean Sci. 28: 976-980 (Abstract in English).

Wang GL and Fang HJ (2002). Plant gene engineering principle and technology. 2nd edn. Science Press, Beijing.

Wang JK (2009). Inclusive composite interval mapping of quantitative trait genes. Acta Agro. Sin. 35: 1-7 (Abstract in English).

Wang XD (2005). Construction of a mapping population with quality traits and analysis of SSR markers linked to QTLs for seed protein and oils content in soybean. Graduate thesis. Sichuan Agricultural University, Sichuan (Abstract in English).

Watanabe S, Hideshima R, Xia Z, Tsubokura Y, et al. (2009). Map-based cloning of the gene associated with the soybean maturity locus E3. Genetics 182: 1251-1262.

Wu XL, Wang YJ, He CY, Chen SY, et al. (2001). QTLs mapping of some agronomic traits of soybean. J. Genet. Genomics 28: 947-955.

Yamanaka N, Watanabe S, Toda K, Hayashi M, et al. (2005). Fine mapping of the FT1 locus for soybean flowering time using a residual heterozygous line derived from a recombinant inbred line. Theor. Appl. Genet. 110: 634-639.

Zhou R, Wang XZ, Chen HF, Zhang XJ, et al. (2009). QTL analysis of lodging and related traits in soybean. Acta Agron. Sin. 35: 57-65. 